Research Article

\title{
Data-Driven Approaches for Diagnosis of Incipient Faults in Cutting Arms of the Roadheader
}

\author{
Qiang Liu $\mathbb{D}$, , ${ }^{1,2}$ Songyong Liu $\left(\mathbb{D},{ }^{1}\right.$ Qianjin Dai $\mathbb{D}^{2},{ }^{2}$ Xiao Yu $\mathbb{D}^{1},{ }^{1}$ Daoxiang Teng $\left(\mathbb{D},{ }^{2}\right.$ \\ and Ming Wei $\mathbb{D}^{2}$ \\ ${ }^{1}$ School of Mechatronic Engineering, China University of Mining and Technology, Xuzhou 221116, China \\ ${ }^{2}$ School of Physics and New Energy, Xuzhou University of Technology, Xuzhou 221018, China \\ Correspondence should be addressed to Songyong Liu; lsycumt@163.com
}

Received 2 August 2020; Revised 2 January 2021; Accepted 24 January 2021; Published 8 February 2021

Academic Editor: Felix Albu

Copyright (c) 2021 Qiang Liu et al. This is an open access article distributed under the Creative Commons Attribution License, which permits unrestricted use, distribution, and reproduction in any medium, provided the original work is properly cited.

\begin{abstract}
Incipient fault detection and identification (IFDI) of cutting arms is a crucial guarantee for the smooth operation of a roadheader. However, the shortage of fault samples restricts the application of the fault diagnosis technique, and the data analysis tools should be optimized efficiently. In this study, four machine learning tools (the back-propagation neural network based on genetic algorithm optimization, the naive Bayes based on genetic algorithm optimization, the support vector machines based on particle swarm optimization, and the support vector machines based on dynamic cuckoo) are applied to address the challenge in the IFDI of cutting arms. The commonly measured current and vibration data cutting arms are used in the IFDI. The experimental results show that the support vector machines based on dynamic cuckoo outperform the other methods. Besides, the performance of the four methods under different operating conditions is compared. The fault cause of cutting arms of the roadheader is analyzed and the design improvement scheme for cutting arms is provided. This study provides a reference for improving the fault diagnosis of the roadheader.
\end{abstract}

\section{Introduction}

The roadheader is an important production equipment in coal mining, and its operation should be monitored in real time to ensure safe and efficient mining production [1-3]. The armtype roadheader is an integrated operation machine integrating cutting, walking, loading, and dust extinguishing for roadway tunneling in the coal mine. To realize the remote monitoring of a roadheader and improve the efficiency and the safety of the roadheader, the monitoring of cutting arms should be performed in real time. Due to complex occurrence conditions of coal seams, the sudden change of coal seam hardness is often encountered in the work of the roadheader, leading to the abnormal working state of cutting arms, and even the failure of the cutting teeth, the fracture of the reducer gear, the unbalance of cutting motor rotor, and other faults. If these problems were not timely discovered, the safety accident of the roadheader will be caused. Considering the complexity and maintenance demand of the roadheader, the reliability, diagnosability, and maintainability of the systems should be improved in the life cycle management of machines, namely, the control, fault diagnosis, prediction and maintenance, and other health management which should be strengthened in the life cycle. Therefore, a fast and accurate fault diagnosis method suitable for cutting arms is urgently required to realize the intelligent monitoring of a roadheader.

1.1. Related Work. Studies on the status monitoring of manipulator structures or similar structures have been widely conducted. Through the multisensor neural fuzzy theory, Lang et al. designed a fault diagnosis system for an automated industrial fish cutting machine. Signals of sound, vibration, and visual were obtained by microphone, accelerometer, and digital CCD camera, respectively, and the fast Fourier transform was used to convert the signal into the frequency domain [4]. Caccavale et al. developed a joint actuated fault diagnosis method for the robotic arm. The radial basis function neural interpolator was used to interpolate the unknown faults, and the weight of the neural 
interpolator was adaptively adjusted online [5]. Capisani et al. proposed a model-based fault diagnosis technology to identify manipulator actuator and sensor faults. The highorder sliding mode unknown input observer was used to detect actuator faults, which could provide the necessary redundancy for analysis [6]. Grigoriev et al. studied the tool diagnosis problem and the tool residual life prediction algorithm, which was programmed in the parts processing based on siemens. The real-time working state of the tool was monitored and the healthy state of the tool was calculated to realize control of the NC system [7]. To reduce the maintenance time of the machine, Gangadhar et al. collected the vibration signal of the machine tool by the accelerometer and diagnosed the fault of the machine tool. The machine learning technology based on decision tree was used to monitor the machine tool status online, with an accuracy rate of $89.38 \%$ [8]. To detect the faults of the multi-DOF robot arm, Piltan et al. established a stable Arx-Laguerre fuzzy PC-derivative observation system, in which torque signal and joint variable signal were used to identify the sensor and actuator faults [9]. To improve the reliability of the robot system, Cho et al. put forward a fault detection algorithm without using the neural network of the robot physical model to obtain an accurate robot model. Through the collection of torque signal and the process of data in the neural network, effective fault detection and diagnosis were realized [10]. Serving as a reliable tool for fault detection of DC motor, the convolutional network also can be adopted to solve the problem of fault detection and identification of incipient faults with current data [11].

In the abovementioned studies, there are two methods for fault diagnosis: physical model-based method and datadriven method. Since the working environment is complex and changeable for the large equipment, an accurate physical model is difficult to be established. Besides, the data-driven method may find the fault earlier in the application of the digital twin technology, which can largely reduce the loss of the machine. Therefore, the data-driven method has been widely used [12, 13], and new optimal strategies have been designed to improve traditional algorithms [14-16]. Figure 1 shows the application scenario of digital twin technology in the operation and maintenance of a roadheader. In the digital twin technology, many functions such as fault diagnosis, performance prediction, and design optimization can be realized. As a result, the fault can be found timely and the equipment has a better performance.

At present, support vector machines (SVM), backpropagation (BP) neural network, and naive Bayes are common data-driven methods [17, 18]. The data-driven method includes three steps: (1) the sensor signals are accurately collected; (2) the data preprocessed by these signals are classified by machine learning algorithm; (3) the status monitoring and fault judgment of the equipment are carried out in real time.

However, the shortage of fault samples restricts the application of data-driven methods. Generally, a lot of prior knowledge and sufficient learning samples are required in most data-driven methods. For the mechanical equipment system, especially the large equipment, once the fault occurs, there are only few fault samples, but huge economic losses. Consequently, these excellent theoretical diagnostic methods are difficult to perform well in practical application. Based on the structural risk minimization principle, the SVM theory is a new set of theory systems designed for the machine learning with a small size of samples. Different from the traditional statistical learning theory, the optimal solution can be obtained from limited samples by using the SVM method. Therefore, the SVM method may be suitable for the IFDI of cutting arms.

Many scholars have studied the application of SVM. Bashiri et al. applied the SVM algorithm to the water quality monitoring and used the PCA as the data pretreatment method. Finally, the classification accuracy of the SVM method reached 98\%, and the pattern of water eutrophication can be successfully identified [19]. Kang et al. classified hyperspectral images by the PCA and SVM. Edge holding filters with different parameter settings were applied to the image under consideration, standard edge-preserving features (EPFs) were constructed, and the resulting EPFs were stacked together. PCA-EPFs were classified by the SVM classifier [20]. To improve traditional methods of prostate cancer screening and classification, Siqueira et al. adopted a combination of genetic algorithm (GA) and SVM with the Fourier transform mid-infrared spectroscopy. The results showed that the new method greatly compensated for the defects of the traditional methods [21]. Jung et al. used the PCA-assisted SVM method for reliable channel reservoir characterization. The main geological characteristics of reservoir models were figured out by PCA, and the parameters were projected into a two-dimensional plane by multidimensional scaling based on Euclidean distances [22]. Alickovic and Subasi used the ensemble SVM method to classify the sleep stages. The signals taken from the electrode were denoised using multiscale PCA, and the most informative features were extracted by the discrete wavelet transform [23]. Arsalane et al. developed PCA-SVM algorithms for the rapid prediction and identification of beef freshness. PCA was used as a predictive model and SVM was used for beef classification. The MATLAB software was used to analyze the projection model of PCA, and three groups of projections representing the beef freshness during the cold storage were obtained [24]. Since traditional SVM methods were not suitable for computing different features of dataset features, Varatharajan et al. used the SVM model and weighted kernel method to classify more features from input signals. Feature selection and discretization level can affect the classification accuracy of the SVM [25]. Tsai and Chen examined the combination of two different sequences of feature selection and discretization with SVM classifiers in terms of classification accuracy and computing time [26]. Wang et al. proposed a stacked sparse autoencoder-based network with the SVM and the principal component analysis (PCA) method to improve the accuracy of fault diagnosis in power systems. To improve the performance of power system fault diagnosis, a new method was adopted, in which PCA was used to process the characteristics after stacked sparse autoencoder training, and the classifier of deep learning was changed from softmax to SVM with the 


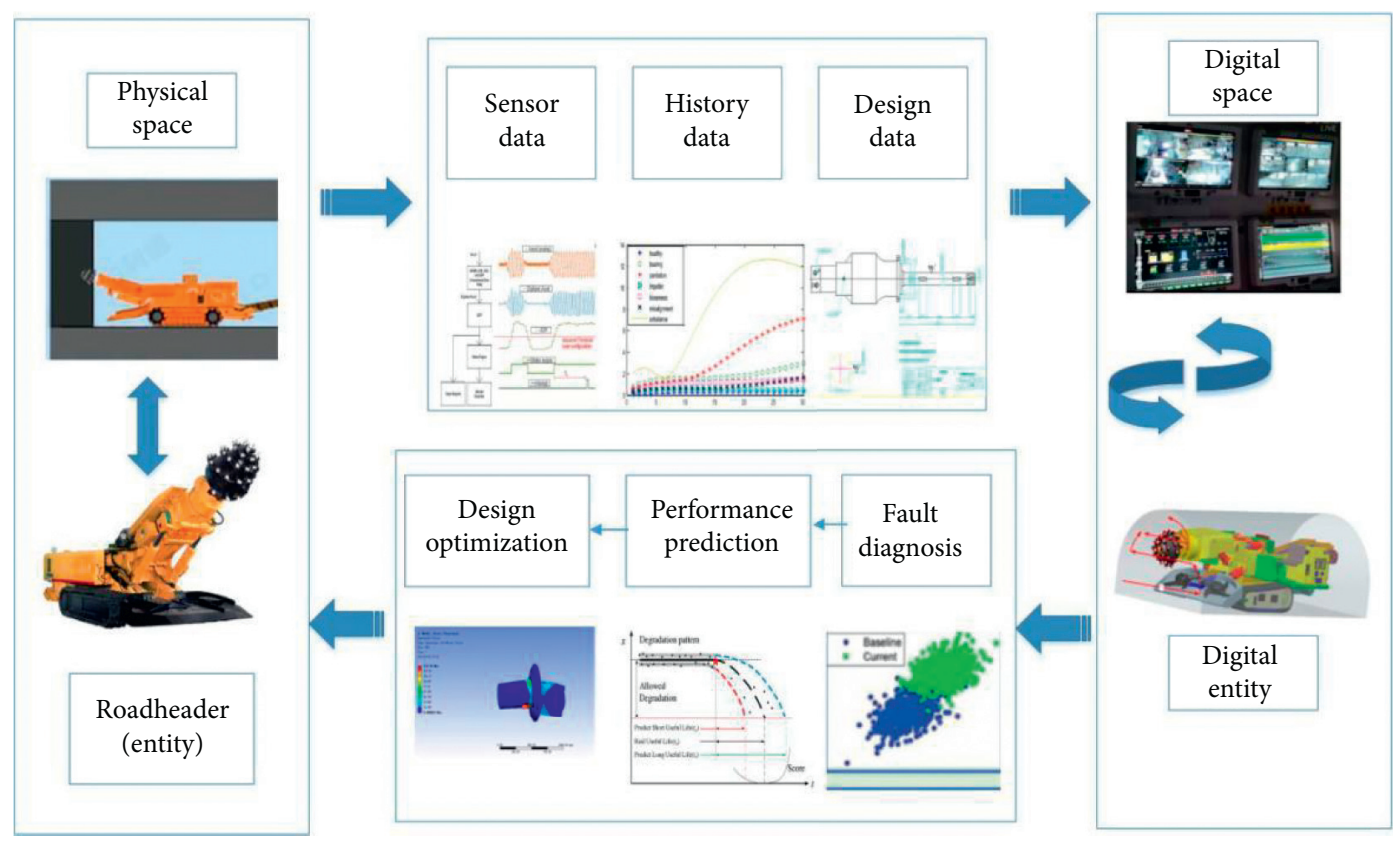

FIGURE 1: Application of digital twin technology in operation and maintenance of the roadheader.

Gaussian kernel [27]. Mathew and Kumar applied the multilinear PCA with SVM in the big data disease diagnosis [28]. Lazakis et al. studied an SVM-driven method for the state estimation of ship systems to assist the dynamic detection of early faults of ship machinery and reduce the ship downtime [29]. Rudsari et al. analyzed the fault of the HV circuit breaker with an improved SVM classifier. The kernel function is first used to transfer the feature vectors to a higher-dimensional space, and then SVM is used to classify these features. The classification results are greatly influenced by the selection of kernel function [30].

In summary, although many efforts have been made to optimize the pattern recognition model in the SVM, efficiency and accuracy cannot be fully ensured. Besides, the SVM has not been used in the field of mechanical cutting arms status monitoring and fault diagnosis. In this paper, a new SVM algorithm based on hybrid optimization of cuckoo and particle swarm optimization (PCSVM) for fault diagnosis of cutting arms is proposed, which can quickly and accurately identify cutting arms of the roadheader with limited samples. This study provides an idea for unmanned monitoring of the roadheader.

1.2. Contributions. The contributions of this paper are as follows:

(1) The PSO and CS are combined in a new PCSVM method to optimize the SVM. The new PCSVM method is applied to the IFDI of cutting arms and it significantly improves the IFDI capability.

(2) The performance of PCSVM in the IFDI of cutting arms is compared with that of other data-driven approaches, including the BP network based on GA optimization (GAB), naive Bayes based on GA optimization (GANB), and SVM based on particle swarm optimization (PSOSVM).

(3) Signals of vibration and current are collected from experiments as the training and testing samples to verify the effectiveness and practicability of the proposed methods mentioned above.

(4) The fault cause of cutting arms of the roadheader is analyzed and the design improvement scheme for cutting arms is provided. These issues have rarely been reported in previous studies.

This study is organized as follows. The different operating conditions of cutting arms and the analysis tools are introduced in Section 2. The proposed techniques for fault diagnosis under cutting and the noncutting conditions are described in Section 3. The experimental setup and data preprocessing are introduced in Section 4. The results on data collected from real-life cutting arms are presented in Section 5. The conclusions are drawn in Section 6.

\section{Preliminaries}

2.1. Operating Conditions of Cutting Arms. The roadheader is the core equipment of underground excavation and plays a vital role in the safe and efficient production of the coal mine. The cutting process of a roadheader is influenced by many factors, and there are potential risks of software failure and software-hardware interaction fault. Due to complex electromagnetic conditions in the roadway, ultra-wide band signals are vulnerable to interference or occlusion failure; then, the nonlinear and strong interference of the whole electro-hydraulic control system can easily lead to the instability of the cutting process. Rapid and accurate fault diagnosis of cutting arms should be performed to ensure the accuracy of continuous operation; otherwise, the problems 
of overbreak or underbreak are easily caused. Early fault detection helps to develop a timely maintenance plan and avoid greater economic losses. Therefore, a fast and accurate fault diagnosis method suitable for cutting arms is urgently required to realize unmanned and intelligent control of a roadheader.

Figure 2 shows the working state of a roadheader. Cutting arms are connected to the cutting head and the mainframe. The cutting head directly contacts the rock and breaks up the rock, and the vibration of cutting arms is large. When cutting arms are in the cutting state, both the vibration signal and the motor current signal peak value are larger; when it is in the noncutting state, the signal value is smaller. In a word, the state of the cutting arm should be monitored and the cutting arm should be quickly and accurately diagnosed.

2.2. Incipient Fault in Cutting Arms. Due to the sudden change of coal seam hardness encountered in the work of the roadheader, the degradation fault of cutting arms can be caused, that is, the incipient fault. The incipient faults mainly have the following characteristics:

(1) Weak: when the incipient fault occurs in the early stage, the signal is extremely weak and difficult to be detected. These small faults can only be identified after characteristic processing.

(2) Slow: incipient faults have small amplitude and slow development. Due to the difficult detection of faults, serious consequences can be caused over time, e.g., wear faults of the bearing.

(3) Sudden: incipient faults are characterized by rapid transients. When the faults occur in components, the local area may spread to the entire system, resulting in the collapse of the whole system.

There are two types of incipient faults: the incipient abrupt failure and time-varying failure. The difference between incipient abrupt failures and general abrupt failure lies in the gain percent of deviation. Compared with the actual value under normal conditions, the incipient failure signal is quite small. The incipient failure of the current sensor is expressed as follows:

$$
I_{f}=I_{n}(1+\alpha)
$$

where $I_{f}$ is the current value of the failure sensor, $I_{n}$ is the normal current value, and $\alpha$ is a tiny bias constant.

The incipient time-varying failure is a fault with a small amplitude at the initial stage, which may deteriorate over time. The resistance degradation is expressed as follows:

$$
R_{f}=R_{n} e^{-\eta t f},
$$

where $R_{f}$ is the failure resistance value, $R_{n}$ is the normal resistance value, and $\eta$ represents the attenuation index.

Figure 3 shows the time-domain vibration signals of cutting arms. No obvious abnormal signals can be found in the time-domain vibration waveforms, and the incipient failure information hidden in signals is difficult to be detected.

Through the further analysis of Figure 3, the frequencydomain vibration signals of cutting arms are obtained, as shown in Figure 4. In this way, the incipient failure signals can be clearly distinguished from the health condition.

When the roadheader is walking in the underground roadway, cutting arms are in the noncutting state, with small vibration and motor current. When the roadheader stops moving forward and cuts the rock wall, the vibration amplitude and the motor current amplitude are relatively large due to the large reaction force from the rock wall. If cutting arms fail, the signal jumps. At this point, if the signal is accurately collected and inputted into the established fault diagnosis model, the fault can be judged immediately. Therefore, the working state of cutting arms is divided into cutting healthy, cutting fault, noncutting healthy, and noncutting fault.

The cutting part is composed of the cutting head, stretching part, cutting reducer, and cutting motor. As the connection between the cutting motor and cutting reducer is manually assembled, this part is prone to failure. The rotating shaft of the motor and the rotating shaft of the reducer are connected by rolling bearings. The deformation and failure of rolling bearings can result in abnormal working state of cutting arms of the roadheader. Through this accident experiment, the accuracy and timeliness of the recommended method are verified.

2.3. Hilbert-Huang Transform and SVM. The Hilbert-Huang Transform (HHT) includes the empirical mode decomposition (EMD) and the Hilbert time spectrum [31, 32]. Firstly, the signal is decomposed into several inherent mode functions (IMF) by the EMD method. Then, the HHT is applied to each IMF component to obtain the instantaneous frequency and instantaneous amplitude, and then the complete time-frequency distribution is obtained. In this work, the HHT is employed as the feature extraction method.

If the signal is nonstationary and does not meet IMF conditions, it is assumed that any complex signal is composed of some independent IMF components. For signal $x$ $(t)$, the EMD process is performed as follows:

(1) Determine all local maximum and minimum points of the signal.

(2) Interpolation operations are carried out for all maximum and minimum points, respectively, and the average value of the upper and lower envelope $a_{1}$ is calculated. The first IMF component of $x(t)$ is $b_{1}$, and $b_{1}=x(t)-a_{1}$.

(3) If $b_{1}$ is not IMF, the above two steps are repeated as original data for $k$ times, and finally, is obtained. Let $b_{1 k}$ satisfy the condition of IMF, then $b_{1 k}$ is IMF of the first order and marked as $c_{1}$, where $a_{1 k}$ is the mean of the envelopes of $b_{1(k-1)}$.

(4) The residual is calculated as $d_{1}=x(t)-c_{1}$. The above steps are repeated with $d_{1}$ as the original data 


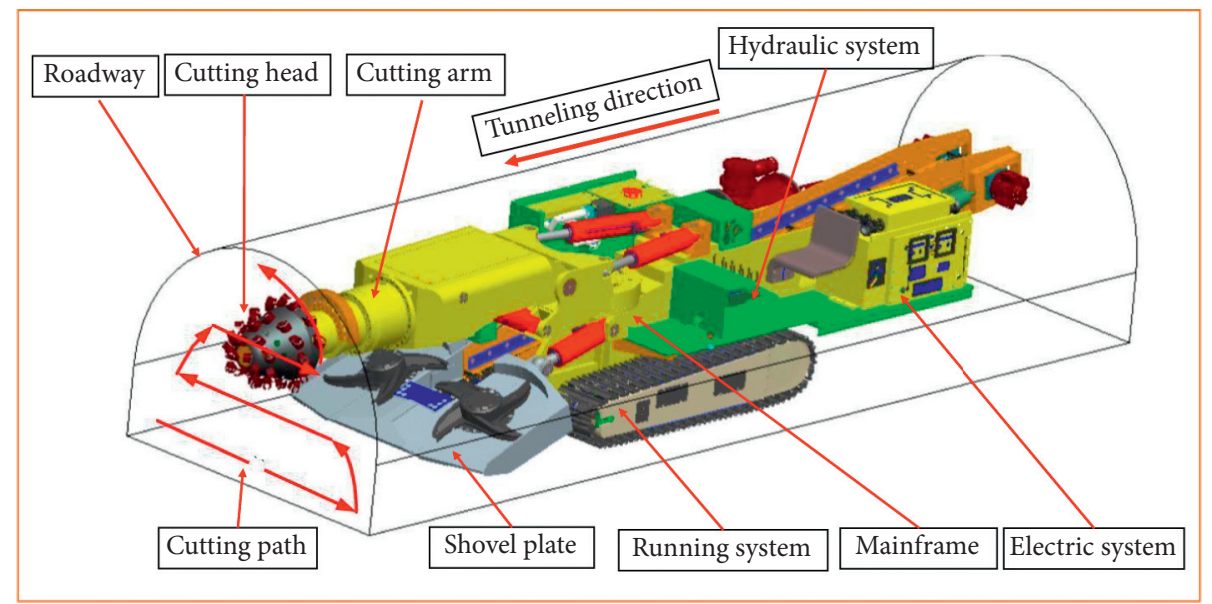

FIgURE 2: Operation diagram of the roadheader.

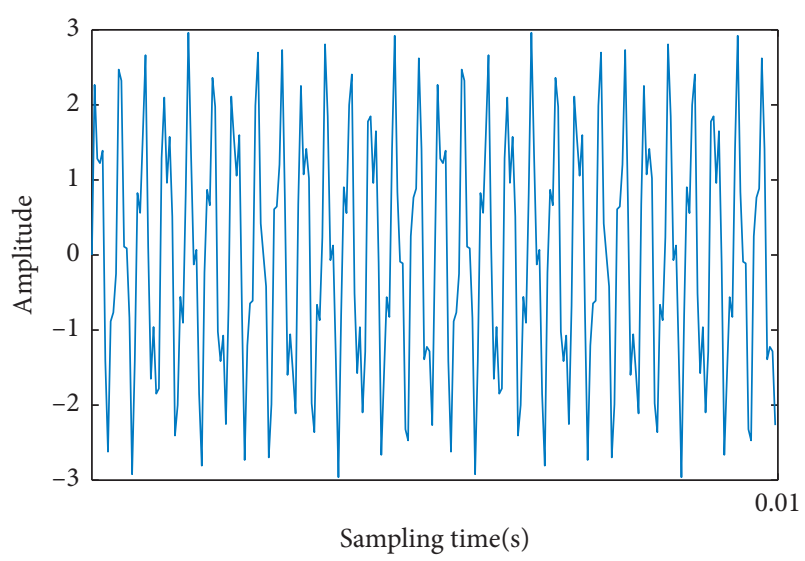

Figure 3: Time-domain vibration signals of cutting arms.

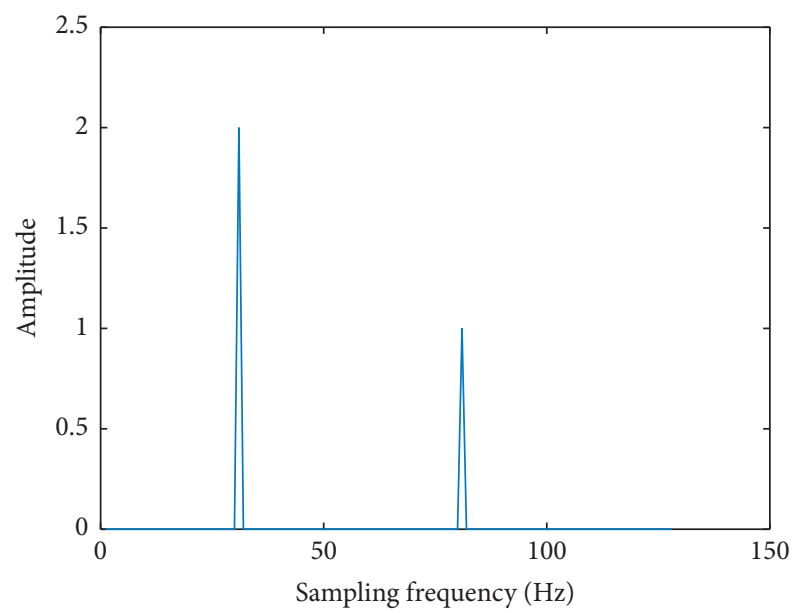

FIgURE 4: Frequency-domain vibration signals of cutting arms.

to obtain the second component of $x(t)$. Similarly, when $d_{n}$ is a monotone function and cannot be used to extract IMF components, the calculation ends and $n$ IMF components are obtained by

$$
x(t)=\sum_{i=1}^{n} c_{i}+d_{n} .
$$

The SVM is a machine learning method based on statistical learning theory. It is a new machine learning method developed on the basis of statistical learning theory, and it is the realization of structural risk minimization principle. The SVM can achieve the purpose of classification and obtain a good statistical law in the case of a small statistical sample size. In this study, the feature is extracted from the HHT of the vibration and current data to classify the operating condition of cutting arms through the SVM. In the next section, the automatic feature extraction and classification from data are described.

Figure 5 shows the implementation of the proposed feature extraction technique. Firstly, the collected vibration signals are decomposed into several different IMFs by EMD. Secondly, the IMFs and the originals are applied to feature generation, and the most sensitive features are selected to construct the feature vector. The cumulative frequency amplitude, which contains frequency features, is expressed by the HHT marginal spectrum across the entire measured time. Thirdly, the low-dimensional feature space is obtained by the PCA in feature dimension reduction. Lastly, the lowdimensional feature set is applied as the input to the classifier.

2.4. BP Neural Networks and Naive Bayes. The BP neural network is composed of the input layer, hidden layer, and output layer, including the model establishment and error correction. According to the training results and expected results, error analysis is carried out, and then weights and thresholds are modified to obtain a model whose output is consistent with expected results step by step [32, 33]. The neural network is composed of adaptive units connected to simulate the interaction between the biological nervous system and the real world. The standard BP algorithm is based on the principle of gradient descent; however, the 


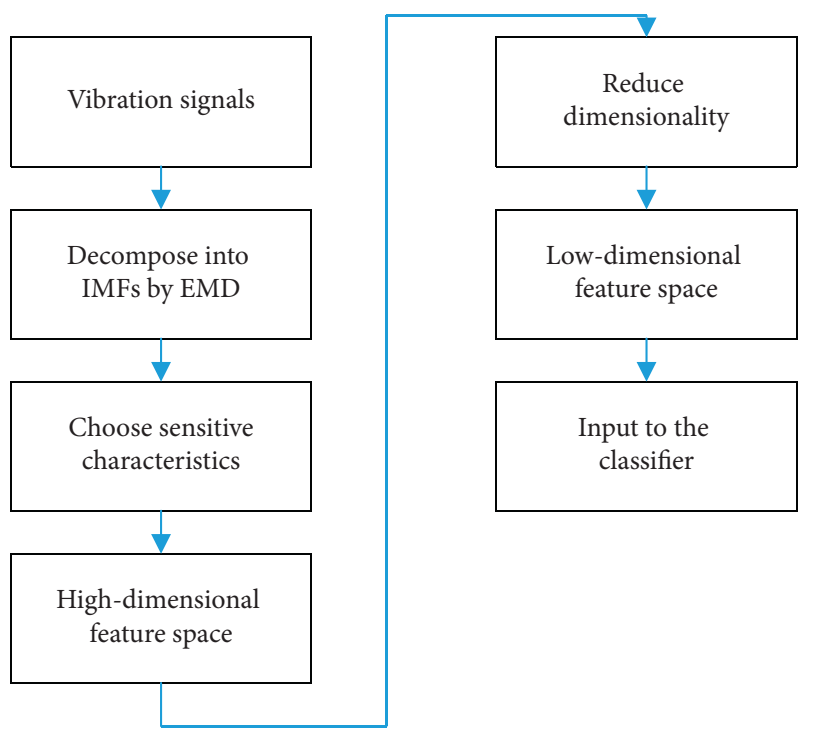

FIGURE 5: Implementation of the proposed feature extraction technique.

gradient descent method has the disadvantages of easily falling into local minimum value and slow convergence speed. Thus, the GA method is proposed to overcome the disadvantage. The individuals can mutate in the GA method $[34,35]$, and the mutation operation is as follows:

$$
a_{i j}= \begin{cases}a_{i j}+\left(a_{i j}-a_{\max }\right) \times f(g), & r>0.5, \\ a_{i j}+\left(a_{\min }-a_{i j}\right) \times f(g), & r \leq 0.5,\end{cases}
$$

where $a_{\max }$ denotes the upper bound of gene $a_{i j}$ and $a_{\min }$ denotes the lower bound of gene $a_{i j}$. Besides, $f(g)$ can be expressed as $f(g)=r_{2}\left(1-\left(g / G_{\max }\right)\right)$, where $r_{2}$ denotes the random number between ; $[0,1], g$ denotes the number of iterations; $G_{\max }$ denotes the maximum number of evolution; and $r$ denotes the random number between $[0,1]$.

Although the basic construction logic is relatively simple, the naive Bayes classification model owns a much faster computing speed than other similar algorithms, with smaller space for classification problems. The naive Bayes classification model also follows the assumption of attribute independence, that is, under the condition of a given category, each attribute is mutually independent [36-38]. Supposing that the sample space $\left\{C_{1}, C_{2}, \ldots, C_{m}\right\}$ has $m$ categories, in which the dataset has $n$ attributes $A_{1}, A_{2}, \ldots, A_{n}$, given a sample of an unknown class, $X=\left(x_{1}, x_{2}, \ldots, x_{n}\right)$, where $x_{i}$ represents the value of the $i$ th attribute of the sample, namely, $x_{i} \in A_{i}$. Based on this, the Bayesian formula can be used to calculate the sample $X=\left(x_{1}, x_{2}, \ldots, x_{n}\right)$ and the probability of class $C_{k}(1 \leq k \leq m)$ is expressed as follows:

$$
P\left(C_{k} \mid X\right)=\frac{P\left(C_{k}\right) P\left(X \mid C_{k}\right)}{P(X)} \propto P\left(C_{k}\right) P\left(X \mid C_{k}\right) .
$$

Although the independence assumption has defects, the naive Bayes classification model has better classification performance under some conditions. Previous studies have shown that the performance of the classifier can be improved through various improvements. The GA optimization of Bayesian algorithm is an improved algorithm based on the Bayesian algorithm combined with the GA [39]. In this paper, the optimal individual retention method in GA is adopted to process the data. The optimal individual retention method can be used to directly retain the individuals with the highest fitness in the population without pairing and crossover.

\section{Methodology}

In the proposed methods, the problem of fault detection and isolation is performed by two steps: procedure-feature extraction and classification. The task of feature extraction is performed with the HHT, while the feature classification is performed with SVM, BP networks, and naive Bayes, respectively. In the classification task, there are four different classes, namely, cutting healthy (C1), cutting fault (C2), noncutting healthy (C3), and noncutting fault (C4). The proposed methods for different operating conditions of cutting arms are described below. Figure 6 shows a generalized framework of the proposed methods.

\subsection{Fault Diagnosis of Noncutting State}

3.1.1. PSOSVM Approach. The PSOSVM approach is firstly presented, i.e., the fault diagnosis based on the extraction of handcrafted features followed by classification. Hilbert spectrum emphasizes the local properties of signals, which has the intuitive physical significance, and avoids the appearance of false frequency components generated by the Fourier transform and wavelet transform. For each IMF $c_{i}$, the Hilbert transformation is performed as follows:

$$
\widehat{c}_{i}=\frac{1}{\pi} \int_{-\infty}^{\infty} \frac{c_{i}(\tau)}{t-\tau} \mathrm{d} \tau .
$$

The analytical signal is expressed as $z_{i}(t)=c_{i}(t)+$ $j \widehat{c}_{i}(t)=a_{i}(t) e^{j \varphi_{i}(t)}$, where $a_{i}(t)$ is the instantaneous amplitude function and $\varphi_{i}(t)$ is the instantaneous phase function. The Hilbert spectrum is calculated as follows:

$$
H(\omega, t)=\sum_{i=1}^{n} a_{i}(t) e^{j \int \omega_{i} \mathrm{~d} t}
$$

After the signal extraction, analog signals are converted into discrete digital signals through the A-D conversion, and then the current state of the device can be judged by the recommended algorithm in this paper. For the state recognition problem of cutting arms, an optimal SVM algorithm is constructed by selecting the appropriate kernel function according to the actual data model. However, the selection of kernel functions and their parameters is empirical with a certain degree of randomness. In addition, a 


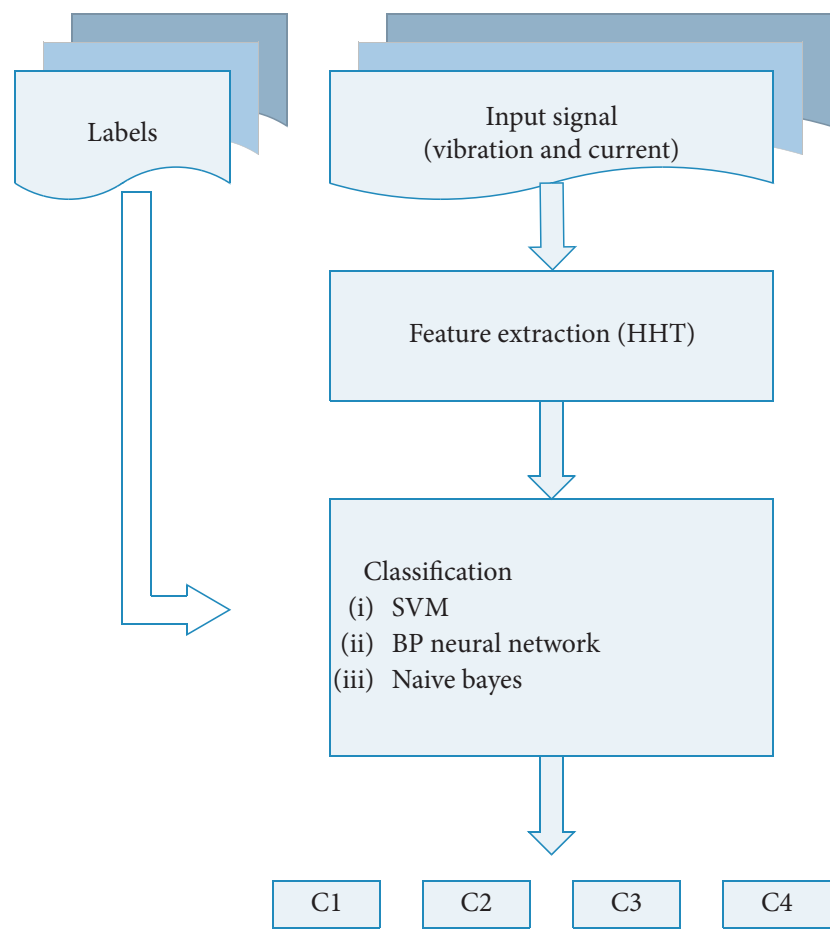

FIGURE 6: A generalized framework of the proposed methods.

combination of PSO and SVM for fault classification can be used.

3.1.2. PCSVM Approach. Particle swarm optimization (PSO) is an intelligent optimization algorithm proposed by Kennedy and Eberhar in 1995. This algorithm has been widely used in the field of engineering optimization because of its simple and fast searching capability of the optimal value [40-42]. However, in the PSO searching process, the current local optimal solution is continuously searched to obtain the final optimal solution, and the local extreme point is easily caused due to the lack of vitality in the later searching stage; finally, the poor calculation accuracy may be caused. The cuckoo search (CS) algorithm proposed by Yang et al. is a new intelligent optimization algorithm inspired by cuckoo nesting and egg-laying behavior. This algorithm has the advantages of self-organization, good parallelism, strong global search ability, and easy to be integrated with other algorithms. In this paper, the combination of PSO and CS is used to optimize the SVM. In the proposed method, when PSO searches the local optimal solution and global optimal solution of each generation, it does not directly enter the next generation but combines with the CS algorithm to continue the search. In this way, the original PSO search range is enlarged and particle search vitality is enhanced, thus effectively making up for the shortage of PSO easily falling into the local extremum point $[43,44]$.

Figure 7 shows the PCSVM calculation process. PSO and CS algorithms are used to find the best penaltyg parameter $c$ and kernel parameter with a high classification accuracy and a high speed.

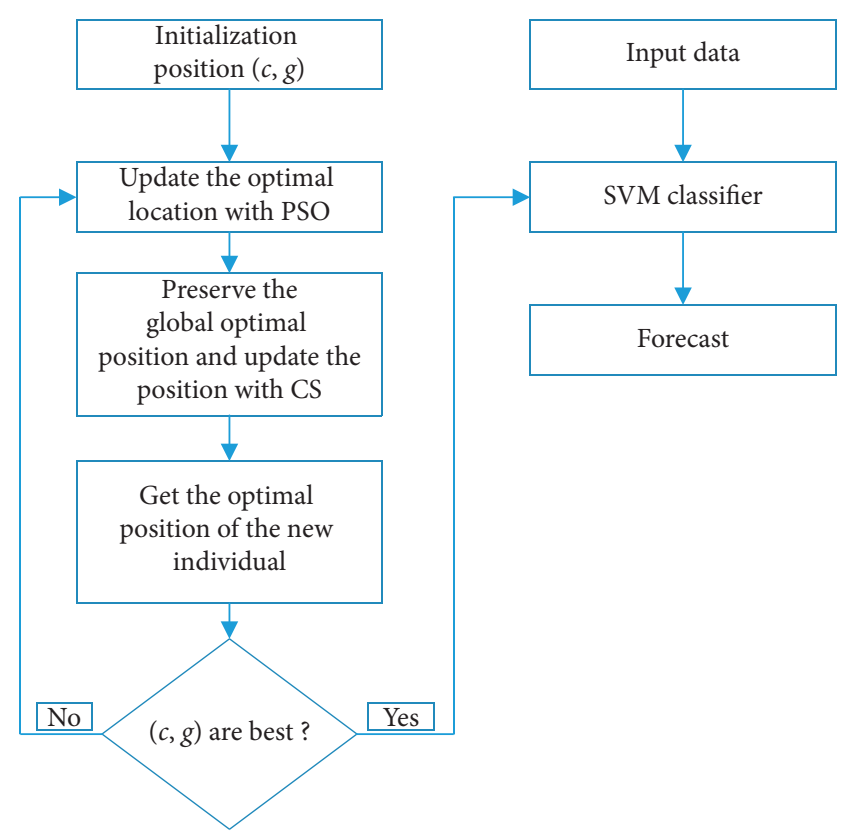

Figure 7: Calculation process of PCSVM.

3.1.3. GAB Approach. The EMD approach is also used for automatic feature extraction, and GAB is used for classification. The GAB is mainly optimized from three aspects: the determination of neural network structure, the GA optimization, and the BP neural network prediction. The GA is used to optimize the initial weight and threshold of the BP neural network, and the optimized BP neural network can be used to effectively predict the function output. The GAB is used to obtain better initial weights and thresholds of the network by GA. The principle of GA algorithm is as follows: the individual is used to represent the initial weight and threshold of the network, and the crossover and mutation operation initiated by individual value in the BP neural network is used to find the optimal individual, that is, the optimal weight and threshold of the BP neural network can be found.

3.1.4. GANB Approach. Lastly, a combination of EMD and naïve Bayes is also used for fault classification. The conditional independence assumption of naive Bayes classifier limits its application to real data. The GA optimization of Bayesian algorithm is an improved algorithm based on Bayesian algorithm combined with the GA so that Bayesian classifier can be applied in fault diagnosis of cutting arms.

3.2. Fault Diagnosis of Cutting State. The characteristic values of vibration signals can be divided into dimensional index and dimensionless index. The dimensional index includes effective value, variance, square root amplitude, mean value, and peak value; the dimensionless index includes kurtosis index, margin index, waveform index, peak value, and pulse index. Effective value kurtosis index and standard deviation have been widely used in 
condition monitoring and fault diagnosis. The intensity of vibration energy is determined by the effective value; the degree of impact is expressed by the kurtosis value, and the degree of deviation from the mean value is expressed by the root of mean square. If the vibration energy suddenly increases under normal conditions, equipment failure may occur. Under normal conditions, the vibration signal is stable when the roadheader is working. Although the fluctuation of fault signal may be very small, its vibration energy, impact, and vibration deviation from the mean value are much larger than those under normal conditions. Therefore, the variance can be used to judge the health degree of the vibration signal state. According to the abovementioned analysis, the variance, effective value, and kurtosis measure are selected as feature vectors of vibration signal $\left[X_{1}, X_{2}, X_{3}\right]$.

As a key cutting part of a roadheader, the cutting motor not only affects the cutting action but also causes the fault of the cutting part directly. Therefore, the running state of the cutting motor should be monitored. The abnormal threephase current of the cutting motor, which is the external direct manifestation of the cutting motor fault, can directly reflect the load state of the cutting motor. Thus, the threephase current of the cutting motor is selected as the feature vector $\left[I_{a}, I_{b}, I_{c}\right]$.

To sum up, the variance of vibration acceleration, effective value, measured peak, and three-phase current of the cutting motor are adopted as feature vectors. Then, a multisensor fusion mode is formed, which can be used to accurately predict the working state of cutting arms.

3.3. Sensitivity Analysis of User-Defined Parameters. To obtain the best performance of these methods, the user-defined parameters are worth investigating. There are two key parameters in $\mathrm{GAB}$, i.e., the number $(N)$ of hidden layers and the number $\left(N_{1}\right)$ of neurons. Obviously, $N$ remarkably influences the computational time of the GAB. The number of levels for $N$ and $N_{1}$ are set as follows: four levels for $N \in\{1,2$, $3,4\}$; four levels for $N_{1} \in\{11,12,13,14\}$. In this experiment, Taguchi's method $[45,46]$ is used to acquire a reasonable combination of two GAB parameters. As a result, $N=1$ and $N_{1}=13$ are obtained according to experimental results.

There are two key parameters in GANB, i.e., the corrected parameter $(\lambda)$ and the prior probability (Pc). The corrected parameter $(\lambda)$ represents the prior knowledge of statistics. In this experiment, the Taguchi's method is used to acquire a reasonable combination of two GANB parameters. As a result, $\lambda=1$ and $P c=0.3$ are obtained according to our experimental results.

There are two key parameters in PSOSVM, i.e., the type parameter $(s)$ and the kernel selection parameter $(t)$. Obviously, the selection of kernel function is determined by $t$. The number of levels for $s$ and $t$ are set as follows: five levels for $s \in\{0,1,2,3,4\}$; five levels for $t \in\{0,1,2,3,4\}$. In this experiment, the Taguchi's method is used to acquire a reasonable combination of two PSOSVM parameters. As a result, $s=0$ and $t=2$ are obtained according to our experimental results.
There are two key parameters in PCSVM, i.e., the type parameter $(s)$ and the kernel selection parameter $(t)$. Similar to the parameters selection of PSOSVM mentioned above, the same results of $s=0$ and $t=2$ are obtained.

3.4. Computation Complexity. The calculation process of these methods mainly consists of two parts: signal processing and pattern recognition. The calculation process is implemented on the same computer with an operating environment of MATLAB. The running time is used to express the complexity of the methods. Table 1 shows the computation time of these methods, and PCSVM performs best among the four methods.

\section{Experimental Setup}

The experimental setup consists of a testbed with threephase asynchronous motor coupled with a gear reducer for changing the load (Figure 8). The fault state is the burn of the connecting bearing of the three-phase motor and the reducer, which is generally caused by poor lubrication and excessive deflection of the shaft. In the signal, the vibration amplitude of cutting arms is suddenly too large and the current amplitude is suddenly changed. The misalignment faults were introduced manually by appropriately adjusting the lubrication of the motor with respect to the shaft connected to the reducer. The current drawn by the motor is measured using a Hall effect sensor that is powered by a regulated DC supply, and the vibration signal is measured using a digital attitude sensor at a sampling frequency of $20 \mathrm{kHz}$. PLC is used for electronically controlling the switches that manipulate the resistive loads and for logging data to a computer.

For each configuration (normal and faults), the motor is operated for one minute, during which 1.2 million measurements of the current and vibration are stored. The acquisition and storage of previous signals are repeated 3-5 times for each configuration. Invalid entries are contained in these data due to sensor effects and external voltage disturbances. These segments are identified and removed in the data preprocessing process. The remaining data are standardized and scaled in the region $[-1,1]$ to speed up the learning process. These examples are constructed for training, validation, and testing of these approaches. Each example is composed of 20 sets of current signals and vibration signals collected, as shown in Tables 2 and 3.

As shown in Table 2, the effective values, kurtosis index, variance, and three-phase current are selected for data preprocessing to training classifiers. In Table 2 , the data of 1-5 lines indicate that the working state is cutting healthy (C1), the data of 6-10 lines indicate that the working state is cutting fault (C2), the data of 11-15 lines indicate that the working state is noncutting healthy (C3), and the data of 16-20 lines indicate that the working state is noncutting fault (C4).

As shown in Table 3, the effective values, kurtosis index, variance, and three-phase current are selected for data preprocessing to verify classification results. The data of $1-5$ 
TABLE 1: Computation complexity of methods.

\begin{tabular}{lcccc}
\hline Process & GAB & GANB & PSOSVM & PCSVM \\
\hline Signal processing (s) & 2.1 & 2.1 & 2.1 & 2.1 \\
\hline Pattern recognition (s) & 6.5 & 5.9 & 5.8 & \\
\hline Total (s) & 8.6 & 8 & 7.9 & 7.2 \\
\hline
\end{tabular}

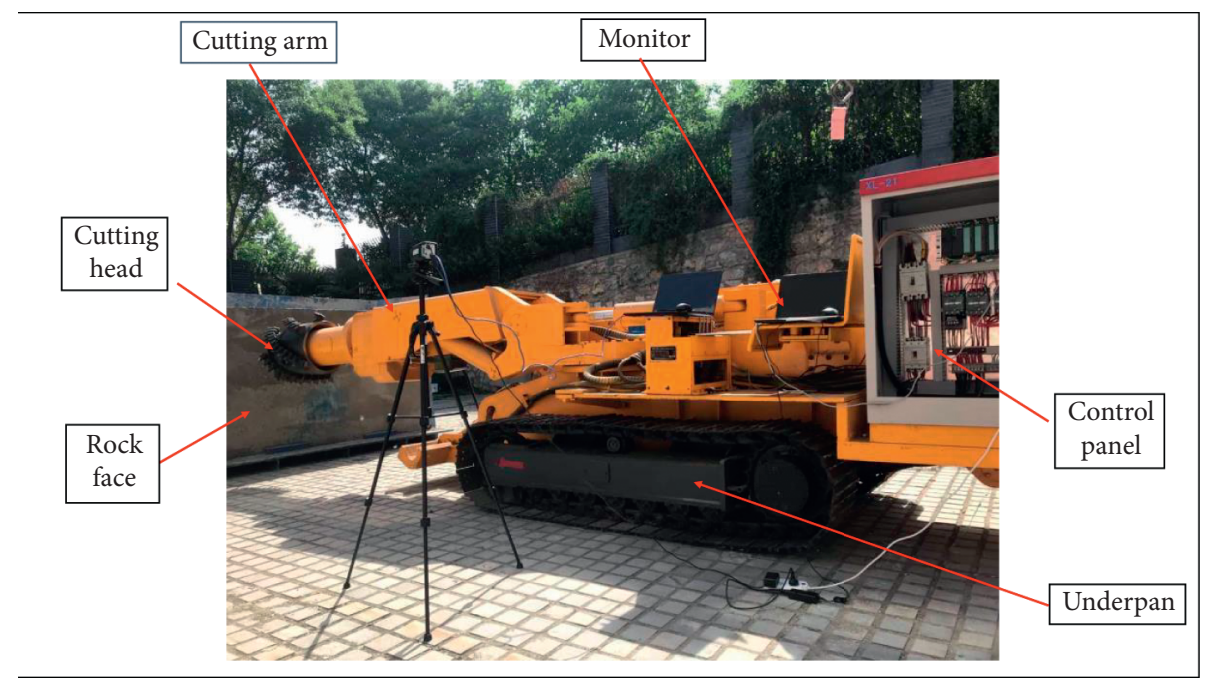

FIGURE 8: Experimental setup.

TABle 2: Sample data.

\begin{tabular}{|c|c|c|c|c|c|c|}
\hline No. & Variance & Effective values & Kurtosis index & Current $A$ & Current $B$ & Current $C$ \\
\hline 1 & 0.0017 & 0.0069 & 3.6634 & 104.30 & 105.00 & 96.80 \\
\hline 2 & 0.0020 & 0.0092 & 2.5883 & 105.00 & 116.30 & 119.30 \\
\hline 3 & 0.0018 & 0.0073 & 2.6638 & 113.30 & 117.80 & 111.80 \\
\hline 4 & 0.0023 & 0.0122 & 2.7874 & 115.50 & 114.80 & 105.80 \\
\hline 5 & 0.0018 & 0.0075 & 3.0039 & 120.80 & 114.80 & 109.50 \\
\hline 6 & 0.0162 & 0.0657 & 23.9742 & 192.53 & 200.18 & 189.98 \\
\hline 7 & 0.0180 & 0.0828 & 23.2947 & 178.50 & 197.63 & 202.73 \\
\hline 8 & 0.0153 & 0.0621 & 32.9706 & 177.23 & 178.50 & 164.48 \\
\hline 9 & 0.0162 & 0.0675 & 27.0351 & 205.28 & 195.08 & 186.15 \\
\hline 10 & 0.0207 & 0.1098 & 25.0866 & 196.35 & 195.08 & 179.78 \\
\hline 11 & 0.0007 & 0.0013 & 1.9910 & 24.75 & 24.00 & 23.25 \\
\hline 12 & 0.0007 & 0.0013 & 2.0277 & 25.50 & 25.50 & 26.25 \\
\hline 13 & 0.0009 & 0.0022 & 2.8295 & 28.50 & 29.25 & 27.00 \\
\hline 14 & 0.0014 & 0.0043 & 2.8934 & 22.50 & 24.00 & 27.00 \\
\hline 15 & 0.0008 & 0.0016 & 2.4496 & 24.75 & 25.50 & 25.50 \\
\hline 16 & 0.0076 & 0.0144 & 22.0464 & 26.25 & 28.50 & 29.25 \\
\hline 17 & 0.0069 & 0.0117 & 18.2493 & 25.50 & 24.00 & 23.25 \\
\hline 18 & 0.0007 & 0.0013 & 2.1622 & 69.83 & 71.66 & 66.15 \\
\hline 19 & 0.0013 & 0.0039 & 2.8532 & 60.64 & 58.80 & 56.96 \\
\hline 20 & 0.0126 & 0.0387 & 26.0406 & 55.13 & 58.80 & 66.15 \\
\hline
\end{tabular}

lines indicate that the working state is cutting healthy (C1), the data of 6-10 lines indicate that the working state is cutting fault (C2), the data of 11-15 lines indicate that the working state is noncutting healthy (C3), and the data of 16-20 lines indicate that the working state is noncutting fault (C4). 
TABle 3: Test sample data.

\begin{tabular}{|c|c|c|c|c|c|c|}
\hline No. & Variance & Effective values & Kurtosis index & Current $A$ & Current $B$ & Current $C$ \\
\hline 1 & 0.0096 & 0.0031 & 3.6624 & 123.00 & 117.00 & 125.25 \\
\hline 2 & 0.0015 & 0.0081 & 2.7834 & 114.00 & 109.50 & 101.25 \\
\hline 3 & 0.0015 & 0.0074 & 2.7603 & 115.50 & 115.50 & 108.00 \\
\hline 4 & 0.0015 & 0.0081 & 2.7889 & 114.75 & 114.75 & 107.25 \\
\hline 5 & 0.0010 & 0.0038 & 2.9848 & 120.00 & 131.25 & 132.75 \\
\hline 6 & 0.0011 & 0.0342 & 4.0286 & 159.90 & 152.10 & 162.83 \\
\hline 7 & 0.0017 & 0.0089 & 3.0617 & 148.20 & 142.35 & 131.63 \\
\hline 8 & 0.0017 & 0.0081 & 3.0363 & 150.15 & 150.15 & 140.40 \\
\hline 9 & 0.0017 & 0.0089 & 3.0678 & 149.18 & 149.18 & 139.43 \\
\hline 10 & 0.0011 & 0.0042 & 3.2833 & 156.00 & 170.63 & 172.58 \\
\hline 11 & 0.0008 & 0.0021 & 3.0072 & 26.25 & 24.75 & 24.00 \\
\hline 12 & 0.0013 & 0.0056 & 2.4134 & 27.00 & 25.50 & 26.25 \\
\hline 13 & 0.0011 & 0.0039 & 2.5398 & 22.50 & 21.75 & 26.25 \\
\hline 14 & 0.0012 & 0.0052 & 3.0413 & 27.75 & 27.75 & 26.25 \\
\hline 15 & 0.0013 & 0.0057 & 2.9891 & 29.25 & 27.75 & 28.50 \\
\hline 16 & 0.0014 & 0.0062 & 2.6547 & 35.10 & 33.15 & 34.13 \\
\hline 17 & 0.0014 & 0.0063 & 3.2880 & 38.03 & 36.08 & 37.05 \\
\hline 18 & 0.0013 & 0.0057 & 3.3454 & 36.08 & 36.08 & 34.13 \\
\hline 19 & 0.0012 & 0.0043 & 2.7938 & 29.25 & 28.28 & 34.13 \\
\hline 20 & 0.0009 & 0.0023 & 3.3079 & 34.13 & 32.18 & 31.20 \\
\hline
\end{tabular}

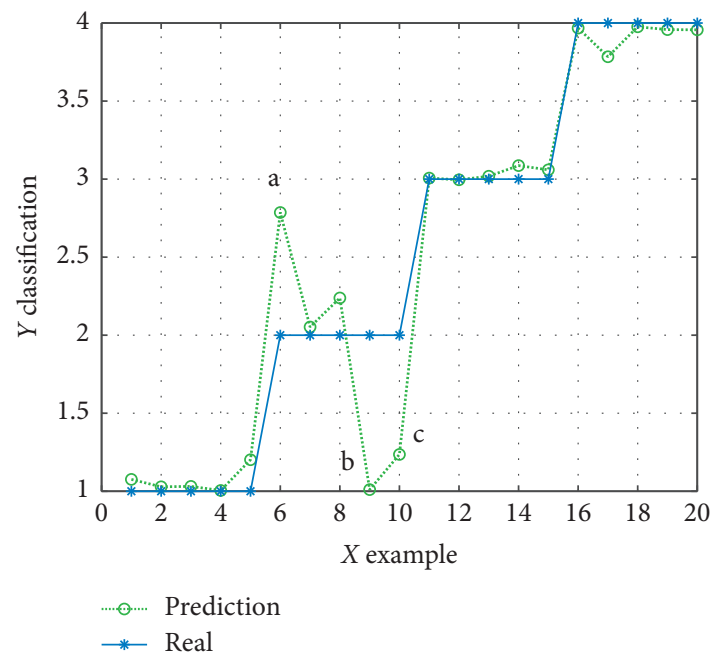

FIgURE 9: The fitting diagram of GAB network.

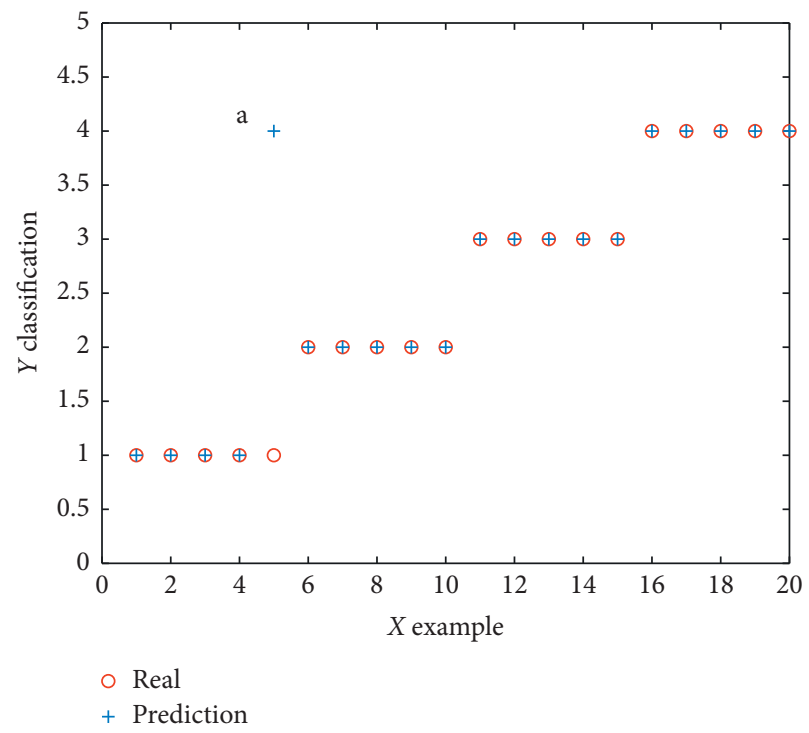

FIgURE 10: The fitting diagram of GANB.

As shown in Figure 11, when the PSOSVM is used to predict the state of cutting arms, there are five prediction errors (points a, b, c, d, and e), and the prediction accuracy is $75 \%$ under the limited samples.

As shown in Figure 12, when the PCSVM algorithm is used to monitor the state of cutting arms, the predicted curve coincides exactly with the real test curve. It means that the monitoring accuracy of the four working states of cutting arms reaches $100 \%$.

As shown in Table 4, the classification accuracy of the GAB, GANB, PSOSVM, and PCSVM approaches is $85 \%$, $95 \%, 75 \%$, and $100 \%$, respectively. In terms of precision, the PSOSVM method performs the worst among these methods. Furthermore, the GANB and GAB approaches have comparable performances in terms of all performance metrics. 


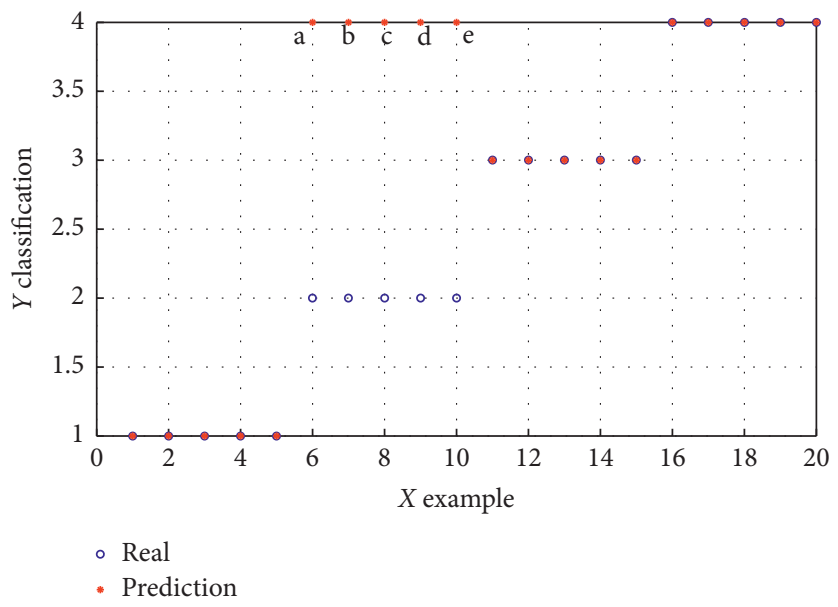

Figure 11: The fitting diagram of PSOSVM.

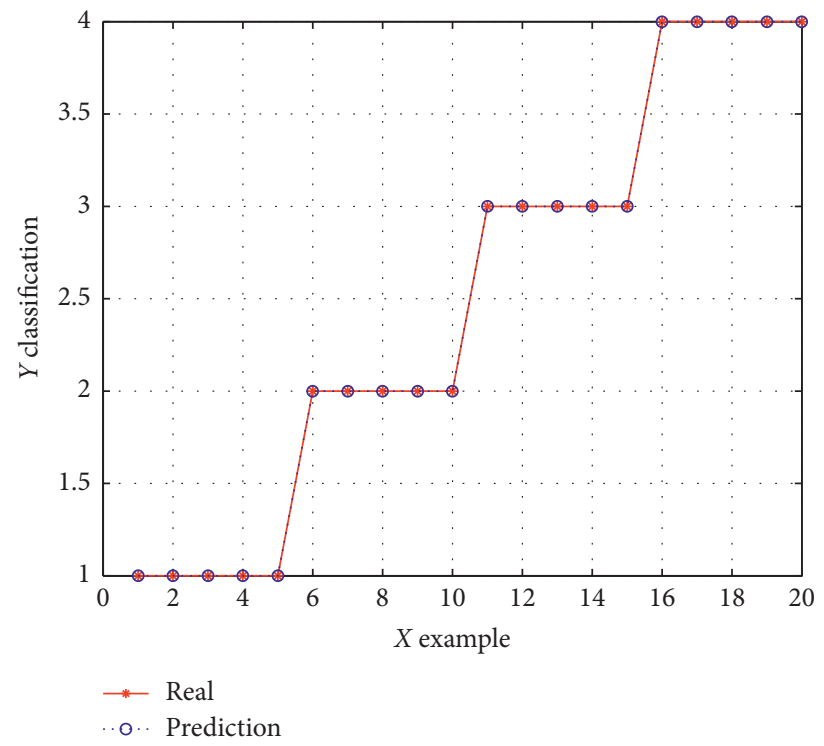

Figure 12: The fitting diagram of PCSVM.

TABle 4: Performance comparison of methods.

\begin{tabular}{|c|c|c|c|c|c|}
\hline Technique & Eigenvector dimension & Training time & Accuracy (\%) & Rate of convergence & Precision \\
\hline GAB & 6 & 1 & 85 & 0.926 & 0.999 \\
\hline GANB & 6 & 0.966 & 95 & 0.927 & 0.925 \\
\hline PSOSVM & 6 & 0.942 & 75 & 0.998 & 0.903 \\
\hline PCSVM & 3 & 0.903 & 100 & 1 & 1 \\
\hline
\end{tabular}

Table 4 clearly suggests that the PCSVM approach has a significant superiority over others with handcrafted features. The best performance of the PCSVM method can be attributed to its adaptive learning ability and classification of fault features, which is not limited to classification based on traditional optimization methods.

5.2. Experimental Analysis. In this experiment, the connecting bearing fault between the motor and the reducer is used as the fault, and the 6218-2RS rolling bearing is used. When the bearing fails, the failure reason is analyzed on-site. It is found that the lack of lubricating oil and excessive offloading of the bearing are the main causes of "burning bearing."

As shown in Figure 13, the main reason for this fault is the lack of lubricating oil, which causes the friction to heat up and burns the bearing. According to the characteristics of burns, they can be divided into mild burns, moderate burns, and severe burns. Moreover, moderate and above 


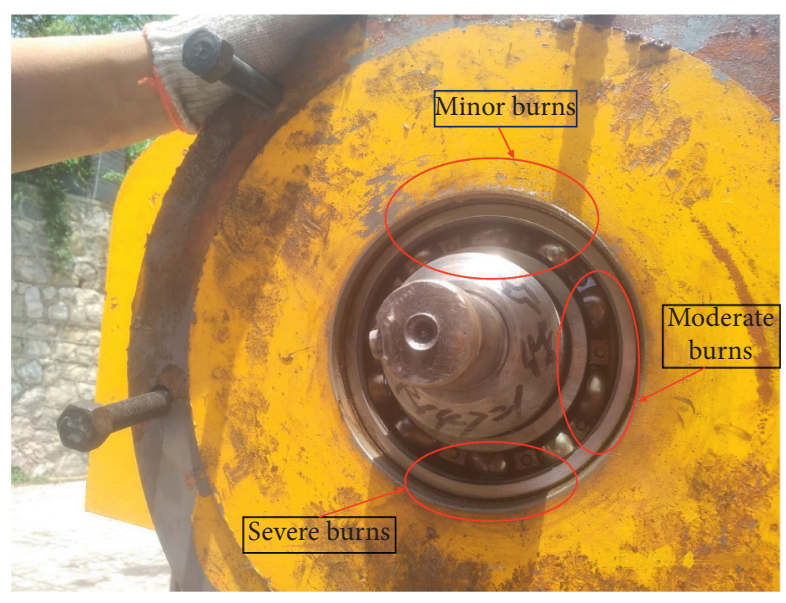

Figure 13: Bearing burn fault.

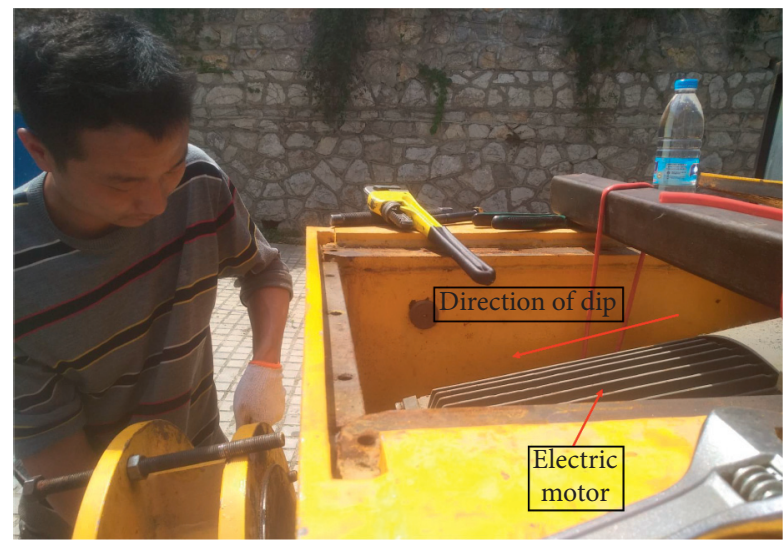

Figure 14: Motor mounting tilt.

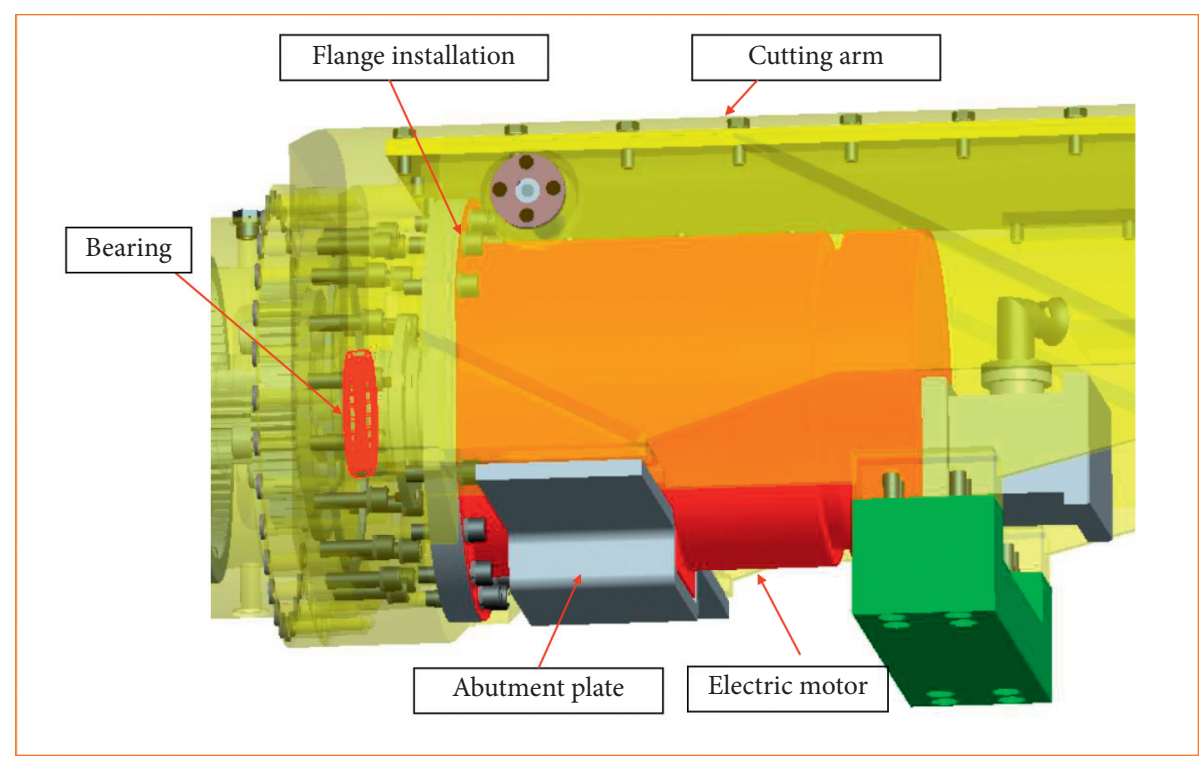

FIgURE 15: Hybrid installation scheme. 
burns are under the lower part of the cage and outer ring. In other words, the bearing also bears too large axial load, which may be the secondary cause of bearing failure. Due to the use of flange installation in the maintenance process of the motor, when the finished flange part is removed and the damaged bearing is taken off, the motor body will tilt, leading to difficulties in bearing replacement. Figure 14 shows the motor tilting.

To reduce the frequency of subsequent failures and facilitate maintenance, the hybrid installation mode of foot mounting and flange mounting should be adopted for the motor. Specifically, an abutment plate can be added at the bottom of cutting arms to connect with the motor base. Figure 15 shows the detailed installation scheme.

When the motor is fixed with the abutment plate and the flange, once the flange is removed and the bearing is replaced, the motor will land on the abutment plate and not be inclined. As a result, equipment maintenance is more convenient, and the service life of the equipment can be increased.

\section{Conclusion}

In this study, the problem of incipient fault detection and diagnosis of cutting arms under different types of conditions is explored. Tools of signal processing (HHT) and statistical machine learning (GAB, GANB, PSOSVM, and PCSVM) are used for pattern extraction and fault classification by the collected data from a DC motor.

The PCSVM approach has the best performance, while the PSOSVM approach has the worst performance. The effectiveness of the four methods is verified through experiments, and the cause of the failure is obtained through the fault mechanism analysis. Furthermore, the design improvement scheme for the hybrid installation is given. In this framework, other variables also can be added to extract more information to improve the robustness of methods. This study provides a reference for status monitoring in digital twin technology.

The approaches can also be extended to other equipment such as the robot arm, the cutting head, the electric motor, and the hydraulic system. In future work, with the collection of more variables, advanced methods can be used to solve the simultaneous identification problem of multiple faults and establish a complete digital twin system for real-time monitoring of device status.

\section{Data Availability}

The training and test sample data used to support the findings of this study are included within the article. The training sample data are shown in Table 2, and test sample data are shown in Table 3.

\section{Conflicts of Interest}

The authors declare that they have no conflicts of interest.

\section{Acknowledgments}

This work was supported by the National Natural Science Foundation of China (no. 51975570), the Natural Science Foundation of the Jiangsu Higher Education Institutions of China (Grant no. 20KJD610002), the project funded by Xuzhou University of Technology (XKY2019202), the Xuzhou Science and Technology Plan Project (KC19244), the Xuzhou Science and Technology Achievements Transformation Plan (KC20203), and the project funded by the Priority Academic Program Development of Jiangsu Higher Education Institutions (PAPD).

\section{References}

[1] S. E. Seker and I. Ocak, "Performance prediction of roadheaders using ensemble machine learning techniques," Neural Computing and Applications, vol. 31, no. 4, pp. 1103-1116, 2019.

[2] A. Salsani, J. Daneshian, S. Shariati, A. Yazdani-Chamzini, and M. Taheri, "Predicting roadheader performance by using artificial neural network," Neural Computing and Applications, vol. 24, no. 7-8, pp. 1823-1831, 2014.

[3] R. Shirani Faradonbeh, A. Salimi, M. Monjezi, A. Ebrahimabadi, and C. Moormann, "Roadheader performance prediction using genetic programming (GP) and gene expression programming (GEP) techniques," Environmental Earth Sciences, vol. 76, no. 16, 2017.

[4] H. Lang, Y. Wang, and C. W. de Silva, "An automated industrial fish cutting machine: control, fault diagnosis and remote monitoring," in Proceedings of the 2008 IEEE International Conference on Automation and Logistics, Qingdao, China, 2008.

[5] F. Caccavale, P. Cilibrizzi, F. Pierri, and L. Villani, "Actuators fault diagnosis for robot manipulators with uncertain model," Control Engineering Practice, vol. 17, no. 1, pp. 146-157, 2009.

[6] L. M. Capisani, A. Ferrara, A. Ferreira De Loza, and L. M. Fridman, "Manipulator fault diagnosis via higher order sliding-mode observers," IEEE Transactions on Industrial Electronics, vol. 59, no. 10, pp. 3979-3986, 2012.

[7] S. N. Grigoriev, V. D. Gurin, M. A. Volosova, and N. Y. Cherkasova, "Development of residual cutting tool life prediction algorithm by processing on CNC machine tool," Materialwissenschaft und Werkstoffechnik, vol. 44, no. 9, pp. 790-796, 2013.

[8] N. Gangadhar, H. Kumar, S. Narendranath, and V. Sugumaran, "fault diagnosis of single point cutting tool through vibration signal using decision tree algorithm," Procedia Materials Science, vol. 5, pp. 1434-1441, 2014.

[9] P. Farzin, M. Sohaib, and J. Kim, "fault diagnosis of a robot manipulator based on an ARX-laguerre fuzzy PID observer," Robot Intelligence Technology and Applications, vol. 751, pp. 393-407, 2017.

[10] C. N. Cho, J. T. Hong, and H. J. Kim, "Neural network based adaptive actuator fault detection algorithm for robot manipulators," Journal of Intelligent \& Robotic Systems, vol. 95, no. 1, pp. 137-147, 2019.

[11] S. Munikoti, L. Das, B. Natarajan, and B. Srinivasan, "Datadriven approaches for diagnosis of incipient faults in DC motors," IEEE Transactions on Industrial Informatics, vol. 15, no. 9, pp. 5299-5308, 2019.

[12] K. Zhong, M. Han, and B. Han, "Data-driven based fault prognosis for industrial systems: a concise overview," IEEE/ 
CAA Journal of Automatica Sinica, vol. 7, no. 2, pp. 330-345, 2020.

[13] X. Wang, X. Liu, and Y. Li, "An incremental model transfer method for complex process fault diagnosis," IEEE/CAA Journal of Automatica Sinica, vol. 6, no. 5, pp. 1268-1280, 2019.

[14] W. Deng, J. Xu, Y. Song, and H. Zhao, "Differential evolution algorithm with wavelet basis function and optimal mutation strategy for complex optimization problem," Applied Soft Computing, vol. 100, Article ID 106724, 2020, In press.

[15] Y. Song, D. Wu, W. Deng et al., "MPPCEDE: multi-population parallel co-evolutionary differential evolution for parameter optimization," Energy Conversion and Management, vol. 228, Article ID 113661, 2021.

[16] W. Deng, J. Xu, X. Gao, and H. Zhao, “An enhanced MSIQDE algorithm with novel multiple strategies for global optimization problems," IEEE Transactions on Systems, Man, and Cybernetics: Systems, pp. 1-10, 2020.

[17] H. Chen, Y. Lu, and L. Tu, "Fault identification of gearbox degradation with optimized wavelet neural network," Shock and Vibration, vol. 20, no. 2, pp. 247-262, 2013.

[18] Y. Fan, C. Zhang, Y. Xue, J. Wang, and F. Gu, "A bearing fault diagnosis using a support vector machine optimised by the self-regulating particle swarm," Shock and Vibration, vol. 2020, Article ID 9096852, 11 pages, 2020.

[19] S. Bashiri, A. Akbarzadeh, M. Zarrabi et al., "Using PCA combined SVM in the classification of eutrophication in Dez reservoir (Iran)," Environmental Engineering and Management Journal, vol. 16, no. 9, pp. 2139-2146, 2017.

[20] X. Kang, X. Xiang, S. Li, and J. A. Benediktsson, "PCA-based edge-preserving features for hyperspectral image classification," IEEE Transactions on Geoscience and Remote Sensing, vol. 55, no. 12, pp. 7140-7151, 2017.

[21] L. F. S. Siqueira, C. L. M. Morais, R. F. Araújo Júnior, A. A. de Araújo, and K. M. G. Lima, "SVM for FT-MIR prostate cancer classification: an alternative to the traditional methods," Journal of Chemometrics, vol. 32, no. 12, p. e3075, 2018.

[22] H. Jung, H. Jo, S. Kim, K. Lee, and J. Choe, "Geological model sampling using PCA-assisted support vector machine for reliable channel reservoir characterization," Journal of Petroleum Science and Engineering, vol. 167, pp. 396-405, 2018.

[23] E. Alickovic and A. Subasi, "Ensemble SVM method for automatic sleep stage classification," IEEE Transactions on Instrumentation and Measurement, vol. 67, no. 6, pp. 12581265, 2018.

[24] A. Arsalane, N. El Barbri, A. Tabyaoui, A. Klilou, K. Rhofir, and A. Halimi, "An embedded system based on DSP platform and PCA-SVM algorithms for rapid beef meat freshness prediction and identification," Computers and Electronics in Agriculture, vol. 152, pp. 385-392, 2018.

[25] R. Varatharajan, G. Manogaran, and M. K. Priyan, "A big data classification approach using LDA with an enhanced SVM method for ECG signals in cloud computing," Multimedia Tools and Applications, vol. 77, no. 8, pp. 10195-10215, 2018.

[26] C.-F. Tsai and Y.-C. Chen, "The optimal combination of feature selection and data discretization: an empirical study," Information Sciences, vol. 505, pp. 282-293, 2019.

[27] Y. Wang, M. Liu, Z. Bao, and S. Zhang, "Stacked sparse autoencoder with PCA and SVM for data-based line trip fault diagnosis in power systems," Neural Computing and Applications, vol. 31, no. 10, pp. 6719-6731, 2019.
[28] J. Mathew and R. V. Kumar, "Multilinear principal component analysis with SVM for disease diagnosis on big data," IETE Journal of Research, 2019.

[29] I. Lazakis, C. Gkerekos, and G. Theotokatos, "Investigating an SVM-driven, one-class approach to estimating ship systems condition," Ships and Offshore Structures, vol. 14, no. 5, pp. 432-441, 2019.

[30] F. N. Rudsari, A. A. Razi-Kazemi, and M. A. Shoorehdeli, "Fault analysis of high-voltage circuit breakers based on coil current and contact travel waveforms through modified SVM classifier," IEEE Transactions on Power Delivery, vol. 34, no. 4, pp. 1608-1618, 2019.

[31] L. Li, J.-F. Tao, H.-D. Yu, Y.-X. Huang, and C.-L. Liu, “Online condition monitoring of gripper cylinder in TBM based on EMD method," Chinese Journal of Mechanical Engineering, vol. 30, no. 6, pp. 1325-1337, 2017.

[32] S. Tyagi and S. K. Panigrahi, "A hybrid genetic algorithm and back-propagation classifier for gearbox fault diagnosis," Applied Artificial Intelligence, vol. 31, no. 7-8, pp. 593-612, 2017.

[33] A. Li, X. Yang, H. Dong, Z. Xie, and C. Yang, "Machine learning-based sensor data modeling methods for power transformer PHM," Sensors, vol. 18, no. 12, p. 4430, 2018.

[34] R. Ahmed, M. El Sayed, S. A. Gadsden, J. Tjong, and S. Habibi, "Automotive internal-combustion-engine fault detection and classification using artificial neural network techniques," IEEE Transactions on Vehicular Technology, vol. 64, no. 1, pp. 21-33, 2015.

[35] L. Eren, T. Ince, and S. Kiranyaz, "A generic intelligent bearing fault diagnosis system using compact adaptive 1D CNN classifier," Journal of Signal Processing Systems, vol. 91, no. 2, pp. 179-189, 2019.

[36] Z. Rasaei and P. Bogaert, "Spatial filtering and Bayesian data fusion for mapping soil properties: a case study combining legacy and remotely sensed data in Iran," Geoderma, vol. 344, pp. 50-62, 2019.

[37] P. R. N. Da Silva, H. A. Gabbar, P. Vieira Junior, and C. T. Da Costa Junior, "A new methodology for multiple incipient fault diagnosis in transmission lines using QTA and Naïve Bayes classifier," International Journal of Electrical Power \& Energy Systems, vol. 103, pp. 326-346, 2018.

[38] N. R. Sakthivel, B. B. Nair, M. Elangovan, V. Sugumaran, and S. Saravanmurugan, "Comparison of dimensionality reduction techniques for the fault diagnosis of mono block centrifugal pump using vibration signals," Engineering Science and Technology, an International Journal, vol. 17, no. 1, pp. 30-38, 2014.

[39] S. Dang, S. Chaudhury, B. Lall, and P. K. Roy, “Tractographybased score for learning effective connectivity from multimodal imaging data using dynamic bayesian networks," IEEE Transactions on Bio-Medical Engineering, vol. 65, no. 5, pp. 1057-1068, 2018.

[40] P. Roy, G. S. Mahapatra, and K. N. Dey, "Forecasting of software reliability using neighborhood fuzzy particle swarm optimization based novel neural network," IEEE/CAA Journal of Automatica Sinica, vol. 6, no. 6, pp. 1365-1383, 2019.

[41] W. Dong and M. Zhou, "A supervised learning and control method to improve particle swarm optimization algorithms," IEEE Transactions on Systems, Man, and Cybernetics: Systems, vol. 47, no. 7, pp. 1135-1148, 2017.

[42] J. Wang and T. Kumbasar, "Parameter optimization of interval type-2 fuzzy neural networks based on PSO and BBBC methods," IEEE/CAA Journal of Automatica Sinica, vol. 6, no. 1, pp. 247-257, 2019. 
[43] J. Zhao, S. Liu, M. Zhou, X. Guo, and L. Qi, "Modified cuckoo search algorithm to solve economic power dispatch optimization problems," IEEE/CAA Journal of Automatica Sinica, vol. 5, no. 4, pp. 794-806, 2018.

[44] Z. Cao, C. Lin, M. Zhou, and R. Huang, "Scheduling semiconductor testing facility by using cuckoo search algorithm with reinforcement learning and surrogate modeling," IEEE Transactions on Automation Science and Engineering, vol. 16, no. 2, pp. 825-837, 2019.

[45] S. Mirjalili, S. M. Mirjalili, and A. Lewis, "Let a biogeographybased optimizer train your multi-layer perceptron," Information Sciences, vol. 269, pp. 188-209, 2014.

[46] S. Gao, M. Zhou, Y. Wang, J. Cheng, H. Yachi, and J. Wang, "Dendritic neuron model with effective learning algorithms for classification, approximation, and prediction," IEEE Transactions on Neural Networks and Learning Systems, vol. 30, no. 2, pp. 601-614, 2019. 